\title{
Una mirada histórica del desarrollo agro-educativo del Municipio El Tuma - La Dalia, Matagalpa
}

\author{
Karla Patricia Dávila Castillo ${ }^{1}$ \\ Enriqueta del Carmen Caldera Reyes ${ }^{2}$ \\ Julio Cesar Laguna Gámez ${ }^{3}$
}

\section{RESUMEN}

El presente ensayo tiene como finalidad abordar la temática sobre la evolución histórica del municipio del Tuma La Dalia, ubicado en el departamento de Matagalpa; tal acción se percibe desde una concepción de dinámica territorial, en sus diversas dimensiones,; además se determinan aspectos puntuales de territorio, desarrollo, educación y sistemas agrícolas; así como indicadores que circundan el desarrollo humano como principal riqueza marcada por el comportamiento y gestión de la institución educativa y su pertinencia rural vinculada al desarrollo. Cabe destacar que el estudio en cuestión, posee una base teórica cimentada en bibliografía internacional, nacional y local, la cual recoge experiencias claves con puntos de vistas diversos que se confluyen en la relevancia que tiene la educación para formación del capital humano y específicamente el joven y que este responda a las exigencias de un mundo local, rural y globalizado. Se enfatiza en el escrito que la aplicación del enfoque de visión conjunta, integradora donde el actor rural, líderes comunitarios, Estado, comunidad académica y empresa privada puedan converger en un mismo sentido, hasta alcanzar la construcción de acuerdos y toma de decisiones institucionales con una visión de territorialidad, que generen desarrollo de capacidades locales.

Palabras clave: Desarrollo, educación, ruralidad, historia, territorio, visión de conjunto.

Recibido: 17 de noviembre de 2016

Aceptado: 16 de diciembre de 2016

1 Docente e investigadora FAREM-Matagalpa, UNAN-Managua. Correo electrónico: kardycas@yahoo.es 2 Docente e investigadora FAREM-Matagalpa, UNAN-Managua. Correo electrónico: carcalre@yahoo.es 3 Docente e investigador FAREM-Matagalpa, UNAN-Managua. Correo electrónico: jlagunagamez@yahoo.es 


\title{
A historical view of the agro-educational development of the Municipality EI Tuma - La Dalia, Matagalpa
}

\begin{abstract}
The present essay aims to address the issue of the historical evolution of the municipality of Tuma La Dalia, located in the department of Matagalpa; Such action is perceived from a conception of territorial dynamics, in its various dimensions; In addition, specific aspects of territory, development, education and agricultural systems are determined; As well as indicators that surround human development as the main wealth marked by the behavior and management of the educational institution and its rural relevance linked to development. It should be noted that the study in question has a theoretical basis based on international, national and local bibliography, which collects key experiences with diverse points of view that converge in the relevance of education for the formation of human capital and specifically young people And that it responds to the demands of a local, rural and globalized world. It is emphasized in the writing that the application of the joint visionary approach, integrative where the rural actor, community leaders, State, academic community and private enterprise can converge in the same direction, reaching the construction of agreements and institutional decision-making with a Vision of territoriality, that generate the development of local capacities.
\end{abstract}

Keywords: Development, education, rurality, history, territory, overview. 


\section{INTRODUCCIÓN}

Al realizar una fijación retrospectiva del sobrevenir de nuestro territorio, es sorprendente el almacenaje de procesos que han transcurrido, sin detenernos en algún momento a reflexionar si la historia demanda más de lo que se ha aportado, preguntarse ¿Ese aporte ha sido tan sustancioso para alcanzar multi-escalaridad o más bien se ha contribuido a generar retrocesos, planteando "cambios para no cambiar"?

En ese sentido, el comentario anterior brinda una pauta del eje rector en este escrito, en el cual se toman dos dimensiones dicotómicas y univocas a la vez, como lo es educación y desarrollo con énfasis en el sector agrario. Si bien es cierto en algún momento se puede tener una visión por separado de ambos aspectos y se establecen inmensas brechas desde las políticas de estado hasta la gestión de sus presupuestos, sin embargo se comparte la frase inspiradora del conspicuo Nelson Mandela (1995) "La educación es el gran motor del desarrollo personal. Es a través de la educación como la hija del campesino puede convertirse en una médica, el hijo de un minera puede convertirse en el jefe de la mina o el hijo de trabajadores agrícolas puede llegar a ser presidente de una gran nación", esa expresión conduce a realizar una serie de planteamientos desde diferentes ángulos, los cuales definen la relación entre ambas dimensiones.

En nuestro contexto se determina la necesidad de abordar la historia del territorio desde un enfoque del desarrollo agro-educativo, presentando una noción que permite explicar el papel de los entornos en que están insertos una serie de componentes entre ellos: Estado, cooperación externa, religioso, partidos políticos, gobiernos municipales, comunidad científica.

Todos ellos asumiendo un papel preponderante como factor de desarrollo; desde una perspectiva a nivel centroamericano, nacional y local, lo que implica resaltar otros factores de carácter político, económico, cultural entre otros: sin perder de vista que todo ello ha dependido en gran medida de la dinámica territorial en que esté cimentado tal desarrollo, en sus diferentes variables a tratar en este ensayo, el desarrollo humano como principio de partida, que implica necesariamente un progreso con equidad centrado en las personas como principal riqueza, el comportamiento y gestión de la institución educativa; y como última variable la contribución que ha generado todo lo anterior a procesos de desarrollo agrario a lo largo de la historia.

\section{DESARROLLO}

\section{Transformaciones que han configurado el territorio a través de la historia}

Para adentrarse a esa mirada histórica-geográfica, se debe destacar el estudio del territorio como elemento clave del proceso de descripción y conocimiento del mismo, para ello es válido reconocer que con el pasar del tiempo se presenta el surgimiento del percepción de territorio y este es 
definido por Ratzel citado por Manzanal, y otros (2006) en el cual afirma Ratzel que como base, en el contexto histórico de la unificación alemana en 1871, y la institucionalización de la geografía como disciplina en las universidades europeas, el término territorio "Es una parcela de la superficie terrestre apropiada por un grupo humano, que tendría una necesidad imperativa de un territorio con recursos naturales suficientes para su poblamiento, los cuales serían utilizados a partir de las capacidades tecnológicas existentes".

El Territorio es el lugar donde desembocan todas las acciones, todas las pasiones, todos los poderes, todas las fuerzas, todas las debilidades, es donde la historia del hombre plenamente se realiza a partir de las manifestaciones de su existencia. La Geografía pasa a ser aquella disciplina que es más capaz de mostrar los dramas del mundo, de la nación, del lugar. (Santos, 2002, p. 9).

La expresión de Santos, ayuda a comprender que para poder dar respuesta y atención a las necesidades que confluyen en el territorio inicialmente se deberá analizar de forma integrada los siguientes aspectos adheridos al territorio como son: el espacio (acciones y objetos) paisaje (concebida como la fotografía o mirada estática del territorio) tiempo (historia).

Por esa razón, las políticas en un territorio como propiedad lo impactan como espacio de gobernancia, y viceversa; que pueden ser representadas como camadas sobrepuestas en que una acción política se desdobla en varios niveles y escalas: local, regional, nacional e internacional. Lo que posibilita valorar que la variedad de relaciones e interrelaciones muestran las múltiples territorialidades, Tal como lo afirma Mançano (2013) "Si se logra comprender cada tipo de territorio como una totalidad multidimensional y organizada en las diferentes escalas y a partir de sus usos particulares nos posibilita entender el concepto de «multiterritorialidad»".

Por ello se inicia básicamente a describir histórica y geográficamente el territorio del municipio Tuma La Dalia, tal como lo aborda Sposito (2004), el cual comparte que desde el concepto que se le da a la ciencia el uso cognitivo y conceptual de territorio con tres aspectos muy presentes en la Geografía "Una natural, otra individual y una tercera espacial. La primera, la concepción naturalista del territorio (territorio clásico), muy conocida, ha justificado históricamente, y aún hoy, las guerras de conquista a través de un imperativo funcional que se sostiene como natural, pero, en verdad, construido socialmente".

Es Importante rescatar en este análisis es que los intereses individuales como los colectivos ya que muchas veces son el detonante que motiva la reestructuración del conocimiento, así como el proceso productivo de ideas, conductas, normas, modelos que rigen en determinado espacio y territorio a un capital social.

Desde el punto de vista histórico el municipio Tuma La Dalia, según el sitio web de la Alcaldía Municipal (2012) considera que la creación data de comienzos de los años 1920, cuando una 
incipiente carretera comunicaba el poblado de El Tuma con la ciudad de Matagalpa, cabecera departamental.

Para entonces el municipio consistía en un caserío disperso, a $3 \mathrm{~km}$ de éste se localizaba el poblado de Carateras, siendo este, el poblado principal. Contaba con una Iglesia Católica, oficina de telégrafos y autoridades policiales. En los años cincuenta del siglo pasado se construyó una trocha para unir los poblados de El Tuma y Carateras. Con la introducción del cultivo del café, se incrementó la concentración de la población en Carateras.

En la medida que se fue incrementando el cultivo del café, se dio la necesidad de ampliar la trocha hasta Cerro Verde en el año de 1960.

En el año de 1976 se construye la carretera Matagalpa - La Dalia - Waslala, con la construcción de la Carretera por La Dalia, se diseñaron las siguientes infraestructuras: Una escuela, una sucursal bancaria y una oficina de telégrafos, lo mismo que el INCEI (Instituto Nicaragüense de Comercio Exterior e Interior) y una tienda de abarrotes de un comerciante proveniente de Matagalpa.

Con el triunfo de la Revolución en 1979, se creó una delegación de gobierno en donde funcionaban las siguientes instituciones: Ministerio de Educación, INSSBI, INE, INRA, Policía Nacional, se construye un centro de salud, una casa comunal y se amplía la escuela. Además de otras organizaciones como ATC (Asociación de Trabajadores de Campo) y ANDEN (Asociación Nacional de Educadores de Nicaragua).

Con la división política administrativa del país, aprobada el 17 de agosto de 1989, es elevado a municipio, el naciente poblado de El Tuma - La Dalia. Se toman en cuenta los datos históricos de La Dalia, sin embargo, no se puede obviar en ningún momento el aspecto geográfico, y en este municipio está organizado territorialmente en una totalidad de 22 barrios y / o comarcas, cabe señalar que la población del municipio es predominantemente rural, representando el $93 \%$ del total municipal versus $7 \%$ de la población ubicado en la zona urbana.

El municipio de El Tuma -La Dalia, es un municipio, joven que tiene escasamente 27 años de existencia como municipio, por decreto presidencial de la Republica de de Nicaragua, que aprovechando esta decisión ejecutiva este territorio se ha desarrollado tanto económica como políticamente, mejorando las condiciones de su población.

El municipio perte $\neg$ nece al departamento de Matagalpa. Tiene una extensión territorial de 651.66 $\mathrm{km} 2$, lo que representa el 9\% del territorio del departamento, que tiene en total 13 municipios. El Tuma - La Dalia, posee una altura de 720 metros sobre el nivel del mar y una posición geográfica de latitud $13^{\circ} 08^{\prime}$ y longi $\neg$ tud $85^{\circ} 44^{\prime}$ (AMUPNOR 2009). 
El Municipio está ubicado a 45 Kilómetros de Matagalpa su cabecera departamental y a 175 Km. de Managua, con su extensión territorial, ocupa el $0.49 \%$ del territorio nacional y con una población aproximada de 62,365 habitantes (INIDE, 2010). El Tuma La Dalia a nivel municipal goza de una división político administrativa constituida por trece ámbitos territoriales o microrregiones, que integran ciento veintiocho comunidades, a nivel catastral existen seis distritos, que integran veintiséis barrios, ubicados en el zona urbana de El Tuma y La Dalia, cinco barrios ubicados en la Zona Suburbana de La Mora, además forman parte de esta división la ciudadela de El Hular (INITER, 2010).

El municipio cuenta con un total de 4,697 productoras y productores agropecuarios individuales: 3,467 son hombres y 1,230 mujeres, propietarios de un total de 4,717 explotacio $\neg$ nes agropecuarias. En una extensión de 83,571 manzanas este municipio tiene el $16 \%$ de las fincas de todo el departamento (CENAGRO, 2013).

En entrevista a pobladores de este municipio, determinan que con la introducción del cultivo del café, entre los años cincuenta y sesenta, se incrementó la concentración de la población en Carateras, muchas familias del departamento de Matagalpa emigraron hacia el poblado, iniciándose para ese tiempo florecientes haciendas cafetaleras. En la medida que se fue incrementando el cultivo del café, se dio la necesidad de ampliar la trocha hasta Cerro Verde, buscando el límite departamental con Jinotega y con el municipio del Cuá Bocay en el año de 1960.

Según INIFOM (sf) con el correr de los años 60 varias familias se fueron asentando en el naciente poblado de La Dalia, el cual como se apunto anteriormente era un empalme de caminos entre Wasaka y la finca llamada La Dalia (distante de esta población a $7 \mathrm{~km}$ al este). La población fue fundada en los terrenos de los señores Abelino Hernández y Francisco Hernández, quienes eran los dueños de las fincas en ese sector. El nombre original de este territorio era la comunidad es San José de Wasaka, la finca fue desmembrándose en pequeños terrenos a viajeros que circulaban por el sector y que se iban asentando al gustarles la región.

Según los resultados del Censo de Población y Vivienda de 1,995, la población del Municipio de El Tuma - La Dalia, creció a un ritmo anual de 8.35 \% desde el año 1,971 hasta el año 1,995. Es una tasa de crecimiento muy alta en comparación con la media anual del país, la cual es de 3.57 \% para el mismo período de tiempo; inclusive es más alta que la tasa de crecimiento Departamental, la cual es de $3.82 \%$ anual.

Para el período 1,995 - 2,000 se proyectó una tasa de crecimiento de $7.37 \%$ anual para el Municipio de El Tuma - La Dalia. Se observa, que el porcentaje de la población rural es mucho mayor que la población urbana (AMUPNOR, 2009). 
La población del municipio es predominantemente rural, en 1995 ésta asciende a 41,011 habitantes, representando el $93 \%$ del total municipal versus $7 \%$ de la población ubicado en la zona urbana con 2, 876 habitantes (AMUPNOR, 2009).

La distribución por sexo a nivel de área geográfica, se aprecia que en la zona urbana predominan las mujeres con un índice de masculinidad de 90 hombres por cada cien mujeres, situación que se revierte en la zona rural donde el índice de masculinidad asciende a 104 hombres por cada cien mujeres, lo que pudiera estar influenciada por la migración rural-urbana mayoritaria de mujeres que se insertan, generalmente, en actividades de servicio (AMUPNOR, 2009).

En la zona rural, según AMUPNOR (2009), la población se encuentra distribuida en 13 ámbitos territoriales conformados en 128 comunidades, con densidades poblacionales, desde $124 \mathrm{hab} / \mathrm{ha}$, en el caso de la comarca La Estrella y muy baja de hasta 58 hab/ha; como es el caso de la comarca Bull Bull. En las zonas donde se concentra la mayor población es en la comunidad de El Guapotal, con una población de 7,705 habitantes, en segundo lugar se encuentran las comunidades del El Coyolar con 6,512 habitantes y Las Veguitas con 5,462 habitantes respectivamente (Estimaciones del DODT, [Departamento de Ordenamiento y Desarrollo Territorial- D.O.D.T. AMUPNOR] basado en proyecciones INIDE, 2005).

\section{La problemática de la educación y su pertinencia rural vinculada al desarrollo}

Para enfocar el punto educativo y muy ligado al desarrollo en todo el sentido de la palabra, se pone de manifiesto el comportamiento de la misma, uno de los derechos fundamentales del hombre es la educación, sin ella no lograríamos la categoría de hombres y mujeres en todo el sentido de la palabra y desde un punto de vista integral: un ser bio sicosocial. Así lo expresa UNESCO (2007), "la respuesta de la educación a la diversidad implica asegurar el derecho a la identidad propia, respetando a cada uno como es, con sus características biológicas, sociales, culturales y de personalidad, que permiten precisamente la individuación de un sujeto en la sociedad".

No obstante la educación a nivel mundial es una necesidad de primera índole y básicamente en el sector rural, donde la mayoría de jóvenes no concluyen la educación media, debido a la cantidad de limitaciones que se han presentado entre ellas: la falta de accesibilidad de los centros educativos, carencia de programas de atención a primaria completa y menos para la educación secundaria, el desinterés mostrado por las familias por atender la educación de sus hijos.

Es oportuno mencionar que la tarea educativa es vista a nivel mundial con premura y de forma especial en la ruralidad, esto es manifestado en los diversos eventos internacionales, inmortalizando el ideal que "la educación constituye un instrumento indispensable para que la humanidad pueda progresar hacia los ideales de paz, libertad y justicia social" (Delors 1996). 
En el contexto actual, se redefine la noción de educación y población rural desde un enfoque de contextualización y ruralización, en ese sentido uno de los cambios que urgen en el mundo moderno es implementar políticas educativas y reformas curriculares innovadoras, esta premura garantizará la formación del capital humano y específicamente el joven, para responder a las exigencias de un mundo local, rural y globalizado, "la educación para la población rural no se define sólo en relación a los sistemas formales educacionales, sino para todos y todas, bajo cualquier modalidad; se entiende como un proceso crítico para superar la pobreza y las desigualdades, en consecuencia para fortalecer la democracia: su correlación positiva con el aumento del Producto Nacional Bruto y de la productividad es innegable, tiene un efecto multiplicador significativo, es un canal de movilidad social" (Machado, 2004 citado por Williamson 2010, p. 4).

El sistema educativo de diversos países y principalmente en Latinoamérica implementa un currículo que no respetar las características contextuales de cada estudiante, se percibe entonces, "El abismo existente entre aquello que el sistema de educación enseña y lo que los educandos realmente necesitan aprender es sencillamente inaceptable.

Esa disfunción educativa es tan perjudicial a nuestra juventud, al sector productivo y al futuro de nuestras naciones que no se puede seguir aceptando teorizaciones, justificaciones y elucubraciones de los “especialistas' que insisten en mantener en los currículos lo superfluo, en vez de reemplazarlo por lo esencial" (Lacki, s.f., p. 5).

La educación es considerada el eje que transversaliza el desarrollo, por tanto en el ámbito mundial se determina clave preparar a los jóvenes para enfrentar los rápidos cambios tecnológicos, económicos y laborales de sus sociedades.

Lucio (2010) refuerza el planteamiento anterior considerando que el desarrollo rural, desde la nueva ruralidad, se fundamenta en principios que debieran irradiar todo el quehacer en las zonas rurales. Algunos ejes de esta nueva perspectiva, en tanto logren desplegarse, esperan contar con la educación como su mejor aliado y dinamizador.

Desde esta nueva lógica, la educación ha de amamantar un desarrollo humano estrechamente ligado a la lucha por los derechos del planeta, tanto desde su haber natural, su biodiversidad, de su diversidad humana expresada en la capacidad y riqueza de cada persona, como de su visión antropológica, social y cultural.

De acuerdo con Lucio (2010) se requiere una educación capaz de enfrentar, desde contenidos, competencias y valores que se orienten, como prioridad, a aportar de forma constructiva y creativa a la superación de las graves brechas de deterioro que sufre nuestra naturaleza. 
La historia de nuestro país refleja el divorcio permanente entre la educación que se proporciona en el sector rural y la realidad, básicamente este divorcio se agudizó en el período neoliberal (19902006) a lo cual el municipio del Tuma La Dalia no estuvo excepto; en donde las necesidades del sector, esta situación tuvo repercusiones desfavorables para que se lograran operativizar los planes, leyes, políticas y metas definidas.

Años después paulatinamente se ha trabajado para hacer realidad el avance educativo, y se intensifica la urgencia de que todas las instituciones y organizaciones sociales se involucren directa y activamente desde la gestión de una educación con base a sus particularidades geográficas rurales, la forma de ser de su gente, con una pedagogía que fortalezca las capacidades cognitivas y psicoafectivas; así como el aprovechamiento de aprendizajes, derechos humanos y de la tierra, la dinámica productiva del agro rural y la al máximo de diversas maneras y en los diferentes niveles.

Lo antes expuesto, obedece a escenarios visibles, entre ellos: la condición de género, la situación económica de la familia, la maternidad adolescente, la inserción temprana al trabajo y la falta de identidad rural, condiciones que producen o generan la migración del campo a la ciudad o hacia otros países; tal como lo reflejan los siguientes datos proporcionados por un estudio realizado por UNICEF (2010) "personas desempleadas 3.3\%, desempleo joven (18 - 29 años): $3.4 \%$, Migración 1.4. \%”.

Como consecuencia, la mayoría de jóvenes rurales en edad para la educación secundaria, tienen diversas ocupaciones tanto remuneradas como no remuneradas que inciden en la viabilidad de sus proyectos de vida, la economía familiar y la sostenibilidad socioeconómica de los sistemas productivos.

A la situación antes mencionada se le suma, entonces, la interrogante ¿Qué educación rural, para qué desarrollo rural? Tal pregunta se ha planteado, pero aún no tiene respuesta concreta, lo que implica un profundo divorcio entre los modelos de desarrollo rural y su educación; la cual ha estado pensada desde un enfoque urbano, aun sabiendo que sus particularidades deben ser respetadas y atendidas como tal.

En el diagnostico realizado por AMUPNOR (2009), encontramos lo siguiente:

La población en edad escolar del municipio de El Tuma La Dalia según INIDE es de 23,888 habitantes en edades de 05 - 21 años; según el Ministerio de Educación de El Tuma La Dalia, actualmente se atiende una población estudiantil 18,405 estudiantes; lo que representa el $77.04 \%$ de la población en edad escolar, este porcentaje sobrepasa la norma a nivel nacional establecida por el Ministerio de Educación. Del total de la población en edad Escolar, 2,259 estudiantes se encuentran en el nivel Preescolar que representan el 12.27 \%; a nivel municipal; la educación 
primaria actualmente tiene una población estudiantil de 12,043 estudiantes y representan el 65.43 $\%$ de la población estudiantil; la modalidad de secundaria según datos del MINED atiende a una población estudiantil de 4,103 estudiantes que representan el 22.29 \%. El municipio atiende a una población adulta de 42 personas con el Ciclo de Educación de Adultos - CEDA, que reciben clases particulares Y que está realizando el Ministerio de Educación en la localidad.

Según datos del MINED; la demanda educativa es atendida por 166 educadoras, 490 docentes y en el personal administrativo cuenta con un personal de 57 personas a nivel urbano y rural; ubicados en los diferentes centros educativos, que atiende las modalidades de Preescolares, Educación Primaria y Educación Secundaria, con una relación estudiantil por docente de 30 estudiantes / profesor; de este análisis se deduce que la relación estudiante - profesor, es aceptable ya que se encuentra por debajo de la norma urbana; que establece que para cada docente la cantidad de estudiantes en atender como mínimo es de 25 estudiantes por docente y como máximo de 40 estudiantes.

Las modalidades que atiende el MINED del municipio de El Tuma La Dalia; se encuentran la Educación Regular y No Regular, la educación regular en el área urbana atiende la modalidad de Preescolar Formal, atendido por dos educadoras, la educación primaria regular urbana y rural es atendido por 145 docentes y la secundaria diurna o regular urbana y rural es atendida por 47 docentes.

En la educación no regular se encuentran las modalidades de Preescolar no formal o comunitario que actualmente está siendo atendido por 164 educadoras; la primaria multigrado extra edad urbana y rural es atendida en la actualidad por 234 docentes, además se cuenta con el programa CEDA en la zona urbana que es atendida por un docente y la secundaria a distancia que funciona los días sábados y domingos en la zona urbana y rural está siendo atendida por 63 docentes.

A nivel general los centros que imparten primaria regular y Multigrado son centros que atienden los turnos matutinos y vespertinos; la mayoría se encuentran en regular estado principalmente en los centros localizados en la zona rural por falta de mantenimiento, el mobiliario es deficitario, falta de servicios de agua, servicio sanitarios, energía eléctrica, bibliotecas y en algunos casos no cuentan con oficinas administrativas, además de áreas que permitan actividades recreativas y educativa apropiadas.

Para cubrir la demanda educativa del municipio, el Ministerio de Educación ha estructurado veinte núcleos Educativos Rurales NER, atendidos en ciento cincuenta y dos centros educativos, ubicados en el área Urbana y Rural del municipio.

En un estudio realizado por el Instituto de Educación de la Universidad Instituto de Educación de la Universidad Centroamericana (IDEUCA) (2012) plantea que el departamento de Matagalpa y 
por consiguiente en el municipio de la Dalia, se caracteriza por presentar problemas como la falta de identidad de la educación rural, debido a la complejidad de ubicación y lejanía que presentan los centros de estudio, esto constituye una brecha histórica, la misma dispersión geográfica conlleva diversas dificultades, en tanto la formación, capacitación, recursos y participación mantienen un perfil bajo.

En este sentido, es válido reconocer que existe una variedad de factores, identificados por IDEUCA y que se consideran como obstaculizadores al desarrollo de un proceso educativo rural de calidad en el departamento, entre ellos:

- Débil formación de los docentes: Por tratarse de zonas rurales, en mayoría, las acciones de formación y capacitación docente son pocas y asistemáticas, con poca incidencia en cambios en el centro educativo y el aula. Ello se refleja en enseñanza rutinaria, sin estrategias dinámicas que propicien la construcción de aprendizajes significativos.

- Débil liderazgo de sus directores: Esta extenuación tiene relación directa con no haber sido preparados para desempeñar esta función, y la poca atención y seguimiento que reciben de las Delegaciones Municipales.

- Dificultades institucionales para el seguimiento del currículum y el proceso de enseñanzaaprendizaje: En este caso no se tratan como el centro de atención de los directores tres aspectos relevantes como es el hecho educativo, el currículum y los procesos de aprendizajes debido a la sobrecarga y concentración de los dirigentes más en el trabajo administrativo.

- Poca interacción entre centros: El aislamiento en que trabajan los centros, les impide compartir experiencias y enriquecer su quehacer, incorporando innovaciones pedagógicas.

- Contenidos educativos débilmente situados en el contexto: Al no disponer de un Proyecto Educativo de Centro y un Proyecto Curricular de Centro que respondan a las características del contexto, los contenidos curriculares son genéricos y débilmente vinculados a los intereses municipales y comunitarios.

- Escuelas centradas en sí mismas: Las características descritas también repercuten para que los centros no se sientan vinculados a la problemática del entorno de pobreza y búsqueda de estrategias de desarrollo, manteniendo los aprendizajes desvinculados de la realidad municipal y comunitaria.

- Por lo general los centros educativos reciben el mismo tratamiento, ya sean rurales, urbanos o ubicados en comunidades étnicas. Básicamente desde su concepción curricular.

En las últimas décadas, tanto en la región centroamericana como en Nicaragua, los gobiernos hacen esfuerzos generados por las múltiples preocupaciones con base en diversos estudios que demuestran la necesidad de atención a la educación ante la nueva ruralidad; los resultados y experiencias en estos estudios han contribuido a que se den transformaciones que generen una nueva perspectiva en pro del ámbito rural. 


\section{Baluartes de sistemas agrícolas en contraposición con la práctica cotidiana}

En nuestro país como política del gobierno de Reconciliación y Unidad Nacional (GRUN), se ha determinado como prioridad y visión del gobierno, el desarrollo integral del ser humano a través de la educación, por ello la creación de espacios de accesibilidad al servicio educativo, potenciando de forma constante el protagonismo de la juventud, la familia, la organización de la población en comunión con la ejecución de acciones importantes para mejorar la educación pública Plan Nacional de Desarrollo Humano (PNDH, 2012-2016).

Sin embargo, aún se presentan importantes retos y desafíos para lograr atender a todos los niños, niñas y jóvenes nicaragüenses. En términos de calidad, el acceso, la igualdad de oportunidades y la prevención de la terminación prematura de la asistencia a la escuela hay mucho que hacer; aun con el desarrollo de estrategias que permiten el aumento significativo en la cobertura educativa en los últimos años.

IDEUCA (2012) emite que "Matagalpa posee gran riqueza agropecuaria y surte al país de exportaciones significativas en café, ganado y otros productos. El $54 \%$ de la superficie de las Explotaciones Agropecuarias son de tamaño mayor a 100 manzanas, las explotaciones de cinco manzanas o menos apenas representan un poco más del $2 \%$ de la superficie. El mayor número de hectáreas se encuentran en el tamaño de 5.01 a 50 manzanas con un 49 \% del total".

Así mismo, IDEUCA, reconoce que este departamento posee su complejidad geográfica, a la vez que su gran riqueza productiva para el país, pero ambas situaciones ameritan una atención cuidadosa y prioritaria en el plano educativo. Una característica de esta región es que su principal producción es generada por la agricultura, donde se destaca los cultivos de café, hortalizas y granos básicos, específicamente el municipio de la Dalia e históricamente ha sido agrario.

El mayor peso de la condición jurídica se encuentra en los productores(as) individuales mientras que los colectivos familiares que ocupan el segundo lugar registran apenas $0.8 \%$. Se experimenta un incremento considerable del $36 \%$ desde 1963 hasta el 2001 en Explotaciones Agropecuarias con régimen de tenencia propia con título.

En relación al aporte del GRUN en esta municipalidad, se cuenta con la implementación de los programas sociales, que se destacan en el PNDH (2012-2016) destacan Usura Cero, Programa de Semilla para Siembra, el Programa de Merienda Escolar, y por su puesto el Programa Productivo Alimentario (PPA).

Así como es evidente la participación de la alcaldía municipal con sus diferentes líneas en la misión de atender la seguridad alimentaria nutricional. Sin obviar la participación de la empresa local.

Después de citar descriptivamente las fortalezas con las que cuenta el municipio en función de 
la atención a la seguridad alimentaria nutricional, se reconocen los esfuerzos que las diferentes entidades realizan, las cuales son numerosos, no obstante, las líneas que se definen son concebidas y ejecutadas de forma dispersa y carente de una cultura evaluativa para darse cuenta si tal o cual proyecto dio resultado.

Sumado a esto, algunas deficiencias como las siguientes: Nuestras familias rurales de Matagalpa, no poseen la cultura de velar por la promoción de nutrición adecuada y saludable de sus miembros, le restan importancia al tema educativo, no es prioridad ni lo exaltan como base del desarrollo del capital social; esto es evidente en el Tuma La Dalia, así como en la mayoría de los municipios, lo que se concreta en una débil calidad educativa, escuelas centrados en sí mismo, lo que repercute para que éstos no se sientan vinculados a la problemática del entorno de pobreza y búsqueda de estrategias de desarrollo, manteniendo los aprendizajes desligados de la realidad municipal y comunitaria.

Al respecto conviene decir, que se reitera las fortalezas que tienen los padres de familia de los centros escolares del Tuma-La Dalia, en su mayoría se dedican a la agricultura de diversos productos nutritivos, otros trabajan en haciendas y poseen conocimientos de prácticas agrícolas y en otros casos son beneficiarios del programa Hambre Cero; a esto se le agrega una fortaleza más y es que los padres tienen patios en sus casas que pueden ser aprovechados para crear huertos familiares, también los centros educativos poseen grandes extensiones de terreno, cabe señalar que ambas fortalezas no son aprovechadas, tal afirmación obedece a que aun teniendo a mano alimentos sustanciosos, éstos no son utilizados para acompañar la merienda escolar, tampoco es aprovechado los espacios productivos para crear huertos escolares; que permitan el sustento del currículo local, la merienda escolar, al desarrollo individual y social; y como primer indicador el aporte a la sostenibilidad del centro.

Se observa entonces que de forma general, el comportamiento educativo- nutricional-agrícolacomunitario, tienen una fuerte incidencia en el desarrollo territorial del municipio en cuestión, el cual se ubica en la zona húmeda de Matagalpa, con un índice de evento de la pobreza extrema del $62.7 \%$, el 26.7\% de desnutrición crónica y el 4\% de desnutrición global según INIDE (2007), aunque sus pobladores se dediquen al cultivo del café, granos básicos y la ganadería; los datos estadísticos expresados son preocupantes.

\section{Oportunidades desde una planificación estratégica con una visión de conjunto}

En el año 2008 la municipalidad en estudio, se convirtió en uno de los pioneros en elaborar y divulgar su Plan Municipal de Ordenamiento y Desarrollo Territorial (PMODT), en él está contenido un breve diagnóstico que hace alusión a la necesidad de atender la educación rural como prioridad en atención de la secundaria en la ruralidad y se está consciente de que ésta ha sido desatendida, reconoce que amerita estar en constante actualización y un continuum educativo 
de modelos o formación política, así como la atención a la educación ambiental, ciudadana, de relaciones humanas y en fin la protección y cuido de la Madre Tierra.

La decisión de realizar un diagnóstico integral forma parte de los acuerdos emitidos por la Asociación de Municipios Productivos del Norte, AMUPNOR (2013) de manera directa, para presentar resultados que se obtengan en sus y demuestren con claridad las fortalezas, limitaciones y potencialidades identificadas, así lo afirma (Renault 2010) "Es un momento de fusión y síntesis del conocimiento crítico de la realidad, teniendo como base el conjunto de informaciones secundarias obtenidas del territorio, complementadas con consultas de campo, discutidas en un proceso dialógico entre técnicos y actores sociales".

En cuanto a la planificación estratégica que cada municipalidad diseñe, este debe responder congruentemente al diagnóstico realizado y dará su aporte para que de forma interinstitucional MINED y municipalidad declaren las acciones concretas de atención a la formación y atención a los docentes, así como el desarrollo del currículo y su vinculación al perfil de ingreso hacia una escuela o técnica o la universidad, las cuales se encargarán de perfilar un profesional con identidad rural comunitaria.

Tal como lo afirma (Godert, 2007) la "prospectiva estratégica" es un proceso de reflexión que permite iluminar la toma de decisiones presentes a partir de posibles situaciones futuras.

Hoy en día la municipalidad del Tuma La Dalia es una de las pocas que participa con firmeza en acciones educativas destinando recursos, brindando capacitaciones a los docentes, estableciendo alianzas y armonización de los territorios y los actores líderes desde la sociedad civil, instituciones privadas y públicas; prueba de ese esfuerzo, hoy el municipio cuenta con la presencia de la universidad estatal y privadas, además es el municipio que a nivel del departamento tiene más apertura de escuelas secundarias en el campo.

En relación a la implementación y evaluación dependerá básicamente de la cohesión del plan elaborado y la concreta distribución de tareas y acciones que cada entidad y cada actor asuma con responsabilidad, así como el liderazgo demostrado por la municipalidad como cabeza ejecutora.

Básicamente el monitoreo y seguimiento estará respaldado por técnicas e instrumentos (Guías evaluadoras), la divulgación constante de los alcances obtenidos y los desaciertos presentados, hacer de la evaluación una cultura y poder de esta forma tomar decisiones consensuadas.

Se considera inminente establecer sinergias con diversos actores que den insumos para incidir en el desarrollo territorial del municipio del Tuma La Dalia, a través de la planificación activa con una cohesión social territorial sólida, desde la práctica de un enfoque holístico, sistémico y de género; con la participación de la familia, con una visión axiológica, una cultura e identidad propia. 
Así establece la invitación Van de Velde, (2016) "Nicaragua hoy tiene las condiciones para asumir el reto de ser ejemplar en cuanto a la 'construcción colectiva de un Sistema Educativo Nacional, basado en actitudes cooperativas', coherente con un proyecto político-pedagógico de nación, sostenible a largo plazo."

\section{CONCLUSIONES}

En forma de conclusiones:

- Para conocer la territorialidad de un espacio se debe iniciar por la historia del mismo, puesto que este descriptivamente te conduce a la evolución y configuración actual.

- La educación es un arma liberadora como lo expresa Freire, no obstante debe ser usada con sumo cuidado para que cubra más que una transversalidad, la profundidad total del progreso cimentado desde el ser.

- El desarrollo humano desde la nueva ruralidad está fundamentado en la educación como su mejor aliado y dinamizador de procesos, especialmente en la creación de una cultura campesina, de amor y apego a la tierra.

- Se necesita en las escuelas ejecución de un currículo local que exprese las particularidades culturales, ambientales antropológicas y económicas específicas que impregnará de sentido y significado en los aprendizajes con un sentido de pertinencia y permanencia y que se enrumbe a la disminución de índice de pobreza para la obtención de territorios homogéneos, descentralizados, competitivos y productivos desde la práctica agrícola de la escuela.

- Los planes estratégicos de cada municipalidad deben reflejar integralidad con un enfoque de visión de conjunto y una especie de cogestión adaptativa de manejo del territorio, con una aproximación multidimensional e interdisciplinaria, dándole un lugar especial a la educación, desde la práctica de acción-investigación y las alianzas de aprendizaje.

- El análisis de los aspectos del desarrollo del territorio, vinculados a la educación y su calidad educativa, son elementos centrales del desarrollo rural, muy importantes definir estrategias de innovación educativa proclives al logro de avances en la creación de capital social.

\section{BIBLIOGRAFÍA}

Alcaldía Municipal (2012) Historia y origen del Municipio del Tuma La Dalia. http://www. alcaldiaeltumaladalia.gob.ni/el-tuma-la-dalia/historia-e-origen/

AMUPNOR (2009). Plan Municipal de Ordenamiento y Desarrollo Territorial de El Tuma La Dalia Marco De Referencia Proyecto Construcción de una Instancia Regional para la Gestión del Territorio. Asociación de Municipios Productivos del Norte - AMUPNOR - Departamento de Ordenamiento Territorial.

CENAGRO (2013). Matagalpa y sus municipios. Censo Nacional Agropecuarios. Nicaragua: INIDE, MAGFOR. 
Delors, J. (1996). La educación encierra un tesoro: compendio. Informe a la UNESCO de la Comisión Internacional sobre la Educación para el Siglo XXI. Paris: UNESCO.

Gobierno de Nicaragua. (2008). Instituto Nacional de Información de Desarrollo . El Tuma La Dalia en cifras. Managua

Gobierno de Nicaragua. (2012). Plan Municipal de Ordenamiento y Desarrollo Territorial del Tuma la Dalia. Managua, Nicaragua, Gobierno de Unidad y Reconciliación Nacional.

Gobierno de Nicaragua. (2012). Plan Nacional de Desarrollo Humano 2012-2016. Managua, Nicaragua, Gobierno de Unidad y Reconciliación Nacional.

Godet. M (2007) Prospectiva Estratégica: Problemas y Métodos. San Sebastian: PROSPEKTIKER

IDEUCA (2004) Educación Rural acompañada de procesos de intervención educativa desarrollado en el período 2000-2003, en el Municipio de extrema pobreza de El Tuma La Dalia Matagalpa. Managua: IDEUCA.

INIDE (2010). Instituto Nacional de Información de Desarrollo. Información de la población del departamento de Matagalpa. Nicaragua: INIDE.

INIFOM. (SF). Ficha municipal del municipio de El Tuma La Dalia, disponible en www.Inifom. gob/Ni/Municipo/dalia/Matagalpa.

Instituto Nicaragüense de Estudios Territoriales (INETER) (2010). Información de las caracterizaciones municipales del departamento de Matagalpa. www.INITER.gob.ni

Lucio, G., R. (2010). Educación y nueva ruralidad. En Andino, P., C.A. (ed.), Activando la Educación. Oportunidad y reto permanentes del país (pp. 307-309). Managua: IDEUCA.

Lacki P. (s.f.). Desencuentros entre educación y desarrollo rural. Brasil, Oficina Regional de Educación de la UNESCO para América Latina y el Caribe.

Lucio, G., R. (2010). Educación para el desarrollo y recuperación del planeta Tierra. En Andino, P., C.A. (ed.), Activando la Educación. Oportunidad y reto permanentes del país (pp. 354-357). Managua: IDEUCA.

Mançano. B. (2013) Ensayo Territorios: teoría y disputas por el desarrollo rural. Universidad Estadual Paulista, São Paulo. http://www.cedem.uh.cu/sites/default/files/116-133.pdf

Mandela. N. (1995) Largo camino a la libertad. Aguilar. ISBN: 9788403013858 https://www. leadersummaries.com/ver-resumen/el-largo-camino-hacia-la-libertad.

Manzanal, M. (2004), Instrucciones, territorio y gestión del desarrollo local rural-local, Rio de Janeiro.

Mesen R. (2009) La situación educativa y ocupacional de los jóvenes rurales y su implicación para la economía familiar, la sostenibilidad del agrosistema y sus proyectos de vida. Ciudad Universitaria Rodrigo Facio, Costa Rica.

Kammerbauer. H. et al (2010) Modelo de cogestión adaptativa de cuencas hidrográficas. Propuesta conceptual basada en la revisión crítica de las experiencias en Honduras y Nicaragua. CATIE.

PREAL (2014) Informe de Progreso Educativo. Calidad y Equidad para el Desarrollo Humano diseñado por el Programa de Promoción de la Reforma Educativa en América Latina y el Caribe. Managua: EDUQUEMOS.

Renault. A. (2010) Guía para la formulación y gestión de planes de desarrollo rural sostenible: un 
abordaje participativo con enfoque territorial. Asunción: IICA, ISBN 13: 978-92-9248-226-8.

Santos, M. (1997). Temica, Espap, Tempo.Globaliza@ e meio temico-cientifico infmmbonal. Siio Paulo, Editora Hucitec.

Sposito (2004), Urbanización, producción y consumo en ciudades medias/intermedias.https:// books.google.com.ni/books?

Van de Velde, H(2016). ¡A construir escenarios, basados en cooperación genuina! http://abacoenred. com/wp-content/uploads/2016/01/La-educaci\%C3\%B3n-en-Nicaragua-2015-2016.pdf. http:// tortillaconsal.com/tortilla/en/node/17355

UNESCO. (2007). Situación Educativa de América Latina y El Caribe: Garantizando la educación de calidad para todos. Santiago de Chile: Oficina Regional de Educación para América Latina y El Caribe.

Williamson, G. (enero, 2010). Todos en el mismo curso. Escuelas Multigrados en la educación rural: reflexiones desde algunas experiencias internacionales. Revista Digital e Rural, Educación, cultura y desarrollo rural. 13 y 14, 1-16. 